\title{
Quasiparticle decay rate of Josephson charge qubit oscillations
}

\author{
Roman Lutchyn ${ }^{1}$, Leonid Glazman ${ }^{1}$, and Anatoly Larkin ${ }^{1,2}$ \\ 1 W.I. Fine Theoretical Physics Institute, University of Minnesota, Minneapolis, Minnesota 55455, USA \\ ${ }^{2}$ L. D. Landau Institute for Theoretical Physics, Moscow, 117940, Russia
}

(Dated: September 16, 2018)

\begin{abstract}
We analyze the decay of quantum oscillations in a charge qubit consisting of a Cooper pair box connected by a Josephson junction to a finite-size superconductor. We concentrate on the contribution of quasiparticles in the superconductors to the decay rate. Passing of a quasiparticle through the Josephson junction leads to the escape of the qubit out of its Hilbert space, and thus determines the decay rate of quantum oscillations. We find the temperature dependence of the quasiparticle contribution to the decay rate for open and isolated systems. The former case is realized if a normal-state trap is included in the circuit, or if just one vortex resides in the qubit; we find exponential suppression of the rate, $\Gamma \propto \exp (-\Delta / T)$, at low temperatures (here, $\Delta$ is the superconducting gap). In a superconducting qubit isolated from the environment $\Gamma \propto \exp (-2 \Delta / T)$ if the number of electrons is even, while for an odd number of electrons the decay rate remains finite in the limit $T \rightarrow 0$. We estimate $\Gamma$ for realistic parameters of a qubit.

PACS numbers: 03.65.Yz, 03.67.Lx, 74.50.+r, 85.25.Cp
\end{abstract}

\section{INTRODUCTION}

Recent experiments with superconducting qubits show promising results, allowing one to observe hundreds of qubit charge or phase oscillations $\frac{1,2,3,4}{}$. A superconducting qubit is essentially a controllable quantum two-level system and can be realized using phase or charge degrees of freedom. Most of the charge qubits use a Cooperpair box (CPB), a small mesoscopic island connected to a large superconducting reservoir via two Josephson junctions. A device with a large superconducting gap, $\Delta>E_{c} \gtrsim E_{J} \gg T$, can be controlled with the gate voltage and magnetic flux, and has only one discrete degree of freedom: number of Cooper pairs in the box. (Here, $E_{c}$ is the charging energy of the island, and $E_{J}$ is an effective Josephson energy of its junctions with the reservoir; $E_{J}$ depends on the flux.) At the degeneracy point the qubit evolves coherently between the ground and excited states, $|-\rangle$ and $|+\rangle$, which are represented by the superposition of 0 or 1 excess Cooper pairs in the box. The frequency of these oscillations is determined by the Josephson energy.

The main difficulty in technological realization of a charge-based superconducting qubit is due to the decoherence present in it. The mechanisms of the decoherence are currently unknown and can be attributed to phonons, two-level systems in insulating barrier, thermally excited quasiparticles ${ }^{5.6}$. In this paper we concentrate on the contribution of the quasiparticles to the decay rate. Quasiparticles having a continuous spectrum are inherently present in any superconducting device and set a fundamental constraint on the coherence time. Quasiparticle "poisoning", first investigated in the context of charge-parity effects in mesoscopic superconductors ${ }^{7.8 .9}$, manifests itself also in the experiments with charge qubits 11.12 .13 .14 .15 . It was reported that even at low temperatures $(\sim 10-50 \mathrm{mK})$ quasiparticles are present in the CPB. If this is the case, Hilbert space of the CPB expands, and the qubit is no longer a simple two-level system. The transient presence of a quasiparticle in the CPB detunes the qubit from the resonant state of Cooper pair tunneling and affects coherent oscillations. In this article we build a quantitative theory of the quasiparticle effect on the charge qubit oscillations.

We consider two regimes which can be realized experimentally: open system corresponding to fixed chemical potential in the reservoir, and isolated system corresponding to fixed number of electrons in the qubit (see Fig.1). The former case allows for a change of the total number of electrons in superconducting parts of the system, and is experimentally realized if a quasiparticle trap, e.g. normal-state part or a vortex, is included in the circuit. The latter case corresponds to a superconducting qubit isolated from the normal-metal environment. Both cases may be relevant in the context of the cavity quantum electrodynamics experiments where the state of the qubit is determined using photon degrees of freedom.

The paper is organized as follows. We begin in Sec. II with a brief overview of the charge qubit before the discussion of the quasiparticle effect on the charge qubit in an open system. In Sec. III we consider the opposite case of the isolated qubit. Finally, in Sec. IV we present simplified results for the quasiparticle contribution to the decoherence rate for the mentioned experimental realizations and discuss how to decrease it.

\section{STATES OF THE QUBIT IN AN OPEN SYSTEM}

Dynamics of the superconducting charge qubit is usually described by an effective Hamiltonian

$$
H_{e f f}=E_{c}\left(N-N_{g}\right)^{2}+H_{J},
$$

where $E_{c}$ is charging energy of the box, $N$ is dimensionless charge of the $\mathrm{CPB}$, and $N_{g}$ is dimension- 

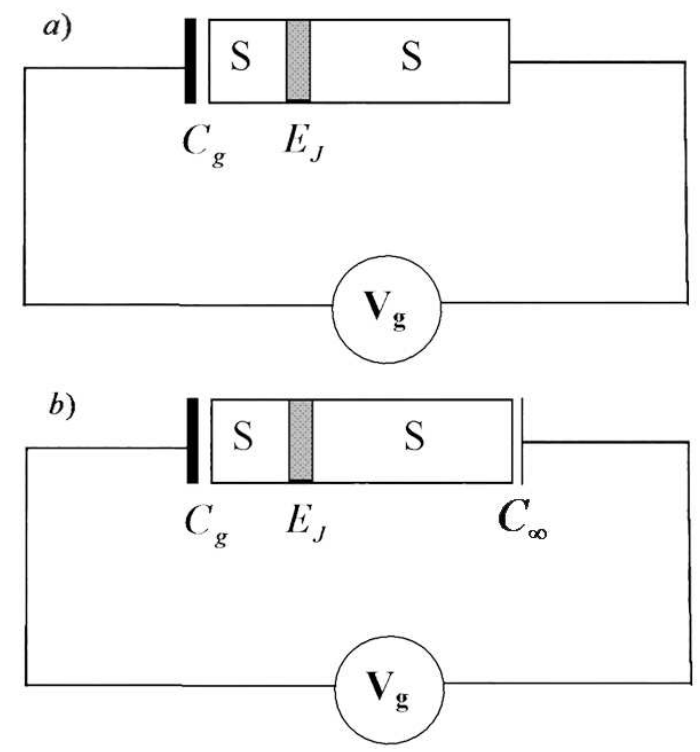

FIG. 1: Schematic picture of the charge qubit in different experimental regimes: a) open system, b) isolated system. The left superconducting mesoscopic island is the Cooper pair box connected via a tunable Josephson junction to the large superconducting reservoir (right). Gate bias is applied through the capacitance $C_{g}$ (assuming that $C_{\infty} \gg C_{g}$ ).

less gate voltage. The Hamiltonian corresponding to tunneling of Cooper pairs, $H_{J}$, is defined as $H_{J}=$ $-\frac{E_{J}}{2}(|N+2\rangle\langle N|+$ h.c. $)$, where $E_{J}$ is effective Josephson energy. In the regime where superconducting gap is the largest energy scale in the system $\Delta>E_{c} \gtrsim E_{J} \gg T$, quasiparticles are usually neglected, and the dynamics of the system is described by the above Hamiltonian, where there is only one discrete degree of freedom - excess number of Cooper pairs in the box. At the operating point, when the dimensionless gate voltage is tuned to be equal to one, $N_{g}=1$, there is degeneracy with respect to charging energy between the charge states $|N\rangle$ and $|N+2\rangle$. This degeneracy is lifted by the Josephson energy, and the states of a qubit are described by the symmetric and antisymmetric superposition of the charge states, $|-\rangle=\frac{|N+2\rangle+|N\rangle}{\sqrt{2}}$ and $|+\rangle=\frac{|N+2\rangle-|N\rangle}{\sqrt{2}}$ with corresponding energies:

$$
\omega_{-}=E_{c}-\frac{E_{J}}{2} \text { and } \omega_{+}=E_{c}+\frac{E_{J}}{2} .
$$

Other charge states have much higher energy, and effectively the Cooper pair box reduces to a two-level system. Once a qubit is excited, quantum oscillations between states $|-\rangle$ and $|+\rangle$ emerge, and the frequency of these oscillations is determined by the Josephson energy $E_{J}{ }^{10}$. The appearance of a quasiparticle with a continuum spectrum provides a channel for relaxation of the qubit. Since quasiparticles are inherently present in any superconducting system, their contribution to the decay rate is intrinsic.

\section{A. Thermodynamic properties of a qubit in an open system}

At a finite temperature, density of quasiparticles is exponentially small, $n \propto \exp (-\Delta / T)$, but the number of quasiparticles in the grain can be of the order of one. It is important to point out that even one unpaired electron can affect the qubit performance. In order to estimate the number of quasiparticles in the grains, one has to account for the huge statistical weight of the states with a single quasiparticle, proportional to the volume of the grain, $N \simeq \sqrt{2 \pi \Delta_{b} T} \nu_{b} V_{b} \exp \left(-\Delta_{b} / T\right)$, where $\nu_{b}$ is normal density of states(per volume), $V_{b}$ volume of the grain. For an isolated dot, the characteristic temperature $\frac{18}{}$ at which quasiparticles appear is $\left(k_{B}=1\right)$

$$
T_{b}^{*}=\frac{\Delta_{b}}{\ln \left(\Delta_{b} / \delta_{b}\right)} .
$$

where $\delta_{b}=\frac{1}{\sqrt{2 \pi} \nu_{b} V_{b}}$ is the mean level spacing in the box, and $\Delta_{b}$ is superconducting gap energy in the box. The appearance of a quasiparticle in the qubit occurs at lower temperature $\widetilde{T}_{b}^{*}$ due to the finite charging energy:

$$
\widetilde{T}_{b}^{*}=\frac{\Delta_{b}-E_{c}+E_{J} / 2}{\log \left(\Delta_{b} / \delta_{b}\right)}=T_{b}^{*}\left(1-\frac{E_{c}-E_{J} / 2}{\Delta_{b}}\right) .
$$

If $T \ll \widetilde{T}_{b}^{*}$, states with odd number of electrons in the box are statistically rare. The probability of finding the qubit in a "good" state (not poisoned by quasiparticles) is important for qubit preparation and is determined by thermodynamics. However, the qubit coherence time is controlled by kinetics, which we study in the next section.

\section{B. Quasiparticle decay rate in the open system}

If an unpaired electron tunnels into the $\mathrm{CPB}$, it tunes the qubit away from the resonant state of Cooper pair tunneling, which leads to the decay of quantum oscillations. At the operating point $N_{g}=1$ it is energetically favorable for a quasiparticle to tunnel to the CPB, since charging energy is gained in such process. Assuming that initially the qubit was prepared in the state with no quasiparticles in the box, the lifetime of the qubit is determined by the time of quasiparticle tunneling to the $\mathrm{CPB}$. In order to estimate this time we use the following Hamiltonian:

$$
\begin{gathered}
H=H_{0}^{\prime}+H_{T}, \\
H_{0}^{\prime}=H_{B C S}^{L}+H_{B C S}^{R}+E_{c}\left(Q / e-N_{g}\right)^{2},
\end{gathered}
$$

where $H_{B C S}^{L}, H_{B C S}^{R}$ are BCS Hamiltonians of the box and superconducting reservoir (see Fig. 1), and $Q$ denotes the charge in the box. The tunneling Hamiltonian 
between two electrodes, $H_{T}$, is defined as

$$
H_{T}=\sum_{k p \sigma}\left(t_{k p} c_{k, \sigma}^{\dagger} c_{p, \sigma}+\text { H.c. }\right)
$$

where $t_{k p}$ is tunnelling matrix element, $c_{k, \sigma}, c_{p, \sigma}$ are the annihilation operators for an electron in the state $k, \sigma$ in the $\mathrm{CPB}$ and state $p, \sigma$ in the superconducting reservoir.

We now consider the lifetime of the qubit in the open system, allowing for a change in the number of electrons in the superconducting reservoir. Assuming that the qubit was prepared in the initial state without a quasiparticle in the box, the time of its coherent evolution is limited by the rate of quasiparticle tunneling to the CPB. In order to calculate the lifetime of the qubit, we have to distinguish between tunneling of Cooper pairs and quasiparticles. To do this, we write the Hamiltonian $H$ in Eq. (5) in the following form:

$$
H=H_{0}+H_{1},
$$

where $H_{0}=H_{0}^{\prime}+H_{J}$, and $H_{1}=H_{T}-H_{J} . H_{J}$ is second order in tunneling amplitude

$$
H_{J}=|N\rangle\left\langle N\left|H_{T} \frac{1}{E-H_{0}^{\prime}} H_{T}\right| N+2\right\rangle\langle N+2|+H . c .
$$

The matrix element $\left\langle N\left|H_{T} \frac{1}{E-H_{0}^{\prime}} H_{T}\right| N+2\right\rangle$ is proportional to effective Josephson energy $E_{J}$, and $H_{T}$ is defined in Eq. (6). Without quasiparticles Hamiltonian $H_{0}$ reduces to Eq. (1).

The quasiparticle tunneling rate is found using Fermi's golden rule and averaging over initial configuration with the appropriate density matrix $(\hbar=1)$

$$
\Gamma=2 \pi \sum_{i, f}\left|\left\langle f\left|H_{1}\right| i\right\rangle\right|^{2} \delta\left(E_{f}-E_{i}\right) \rho\left(\beta H_{0}\right) .
$$

Here, $\rho\left(\beta H_{0}\right)$ is the density matrix for the initial state of the system. The perturbation Hamiltonian accounts for quasiparticle tunneling only, $\left\langle f\left|H_{1}\right| i\right\rangle=\left\langle f\left|H_{T}\right| i\right\rangle$. Thus, Eq. (9) takes into account Cooper pair tunneling exactly while treating quasiparticle tunneling perturbatively. At the operating point, $N_{g}=1$, initial state of qubit is defined by the superposition of 0 and 1 excess Cooper pairs in the box, $| \pm\rangle=\frac{|N\rangle \mp|N+2\rangle}{\sqrt{2}}$; the final state $|f\rangle=|N+1\rangle$ is the state with odd number of electrons in the $\mathrm{CPB}$, corresponding to charge 1e. There are two mechanisms that contribute to the rate of the process $| \pm\rangle \rightarrow|N+1\rangle$ : (1) a quasiparticle tunnels from the superconducting reservoir to the $\mathrm{CPB}$, and (2) a Cooper pair in the box breaks into two quasiparticles, and then one quasiparticle tunnels out into the reservoir. Two corresponding contributions to the total tunneling rate are

$$
\begin{gathered}
\Gamma_{ \pm}=\Gamma_{1 \pm}+\Gamma_{2 \pm} \\
\Gamma_{1 \pm}=2 \pi \sum_{n, p_{j}, k_{i}}\left|\left\langle N+1, k,\{p\}_{n-1}\left|H_{T}\right| \pm,\{p\}_{n}\right\rangle\right|^{2} \\
\times \delta\left(E_{k}-E_{p}-\omega_{ \pm}\right) \rho\left(\beta H_{0}\right)
\end{gathered}
$$

$$
\begin{aligned}
\Gamma_{2 \pm} & =2 \pi \sum_{n, p_{j}, k_{i}}\left|\left\langle N+1, p,\{k\}_{2 n-1}\left|H_{T}\right| \pm,\{k\}_{2 n}\right\rangle\right|^{2} \\
& \times \delta\left(E_{p}-E_{k}-\omega_{ \pm}\right) \rho\left(\beta H_{0}\right),
\end{aligned}
$$

where $\Gamma_{ \pm}$is decay rate for the excited $|+\rangle$or ground state $|-\rangle$ of the qubit, and $\omega_{ \pm}$is defined in Eq. (2). State $\left|+,\{p\}_{n}\right\rangle$, for example, denotes the excited state of the qubit with $n$ quasiparticles in the reservoir with energies $E_{p}=\sqrt{\varepsilon_{p}^{2}+\Delta_{r}^{2}}$

$$
\left|+,\{p\}_{n}\right\rangle=|+\rangle \otimes\left|p_{1}, \ldots, p_{j}, \ldots, p_{n}\right\rangle .
$$

The state $\left|+,\{k\}_{2 n}\right\rangle$ denotes the excited state of the qubit with $n$ broken Cooper pairs in the box, leading to the appearance of $2 n$ quasiparticles with energies $E_{k}=\sqrt{\varepsilon_{k}^{2}+\Delta_{b}^{2}}$

$$
\left|+,\{k\}_{2 n}\right\rangle=\left|+, k_{1}, \ldots, k_{j}, \ldots, k_{2 n}\right\rangle .
$$

In the following, we concentrate on the decay rate of the qubit excited state in the open system, i.e., evaluate $\Gamma_{+}^{\text {op }}$. In order to calculate this decay rate we take into account one-electron processes in the lowest order in quasiparticle density. We assume that in the first contribution to $\Gamma_{+}^{o p}$, Eq. (11), all quasiparticles are in the reservoir and one of them is tunneling into an unoccupied state of the CPB; in the second contribution, Eq. (12), all quasiparticles are in the box and one of them is tunneling out into an unoccupied state of the reservoir. Keeping this in mind, the density matrix for first process can be reduced by tracing out irrelevant degrees of freedom: $\rho_{\mathrm{op}}\left(\beta H_{0}\right)=\operatorname{Tr}_{\{k\}} \rho\left(\beta H_{0}\right)$, and Eq. (11) becomes

$$
\begin{aligned}
\Gamma_{1}^{\mathrm{op}} & =\pi \sum_{n, p_{j}, k}\left|\left\langle N+1, k,\{p\}_{n-1}\left|H_{T}\right| N,\{p\}_{n}\right\rangle\right|^{2} \\
& \times \delta\left(E_{k}-E_{p}-\omega_{ \pm}\right) \rho_{\mathrm{op}}\left(\beta H_{0}\right)
\end{aligned}
$$

Taking into account that only one quasiparticle is transferred through the junction by the action of Hamiltonian $H_{T}$, and performing the sum over momenta in Eq. (15), the contribution of the first mechanism is simplified to

$$
\begin{aligned}
\Gamma_{1}^{\mathrm{op}} & =\pi \sum_{p_{1}, k}\left|\left\langle N+1, k\left|H_{T}\right| N, p_{1}\right\rangle\right|^{2} \delta\left(E_{k}-E_{p_{1}}-\omega_{+}\right) \\
& \times \exp \left(-\frac{E_{p_{1}}}{T}\right)
\end{aligned}
$$

where the exponential factor is the low temperature $\left(T \ll \Delta_{r}\right)$ approximation of the Fermi function. The matrix element $\left\langle N+1, k\left|H_{T}\right| N, p\right\rangle$ can be calculated using the particle conserving Bogoliubov transformation ${ }^{16}$ and is equal to $\left\langle N+1, k\left|H_{T}\right| N, p\right\rangle=2\left(t_{p k} u_{p} u_{k}-t_{k p} v_{p} v_{k}\right)$, where $u_{p}, v_{p}$ are coherence factors

$$
u_{p}^{2}=\frac{1}{2}\left(1+\frac{\varepsilon_{p}}{E_{p}}\right), \text { and } v_{p}^{2}=\frac{1}{2}\left(1-\frac{\varepsilon_{p}}{E_{p}}\right) .
$$

By changing the sum to an integral in Eq. (16) and integrating over $E_{k}$, we get the following expression for $\Gamma_{1}^{\mathrm{op}}$ : 


$$
\begin{aligned}
\Gamma_{1}^{\mathrm{op}} & =\frac{g}{4 \pi} \int_{\Delta_{r}}^{\infty} d E_{p} \frac{\Theta\left(E_{p}+\omega_{+}-\Delta_{b}\right)}{\sqrt{\left(\left(E_{p}+\omega_{+}\right)^{2}-\Delta_{b}^{2}\right)\left(E_{p}^{2}-\Delta_{r}^{2}\right)}} \\
& \times\left(E_{p}\left(E_{p}+\omega_{+}\right)-\Delta_{r} \Delta_{b}\right) \exp \left(-\frac{E_{p}}{T}\right)
\end{aligned}
$$

where $\Theta(x)$ is the step function, $g=\frac{h}{e^{2} R}$ is dimensionless conductance. $\mathrm{R}$ is resistance of the tunnel junction in the normal state

$$
R^{-1}=4 \pi e^{2} \sum_{p, k}\left|t_{p k}\right|^{2} \delta\left(\varepsilon_{p}\right) \delta\left(\varepsilon_{k}\right)
$$

Assuming that mismatch between superconducting gap energies in the box and reservoir is small, $\Delta_{r}-\Delta_{b}+\omega_{+}>$ 0 , which corresponds to most charge qubit experiments, expressions for $\Gamma_{1}^{\mathrm{op}}$ can be simplified. The leading contribution to the decay rate at low temperatures given by the first mechanism is equal to $\Gamma_{1}^{\mathrm{op}}=W\left(\omega_{+}, \Delta_{r}, \Delta_{b}\right)$

$$
W\left(\omega_{+}, \Delta_{r}, \Delta_{b}\right)=\frac{g \sqrt{\Delta_{r}}}{8 \sqrt{2 \pi}} \frac{\left(\Delta_{r}-\Delta_{b}+\omega_{+}\right)}{\sqrt{\Delta_{r}+\Delta_{b}+\omega_{+}}} \exp \left(-\frac{\Delta_{r}}{T}\right)\left[\frac{\Delta_{b}}{\Delta_{r}} U\left(\frac{3}{2}, 2, \frac{\Delta_{r}-\Delta_{b}+\omega_{+}}{T}\right)+2 U\left(\frac{1}{2}, 2, \frac{\Delta_{r}-\Delta_{b}+\omega_{+}}{T}\right)\right]
$$

where $U(a, b, z)$ is the confluent hypergeometric function. At low temperature $\left(T \ll \Delta_{r}-\Delta_{b}+\omega_{+}\right)$asymptotic result for $W\left(\omega_{+}, \Delta_{r}, \Delta_{b}\right)$ is simply given by

$$
W\left(\omega_{+}, \Delta_{r}, \Delta_{b}\right) \simeq \frac{g \sqrt{\Delta_{r} T}}{4 \sqrt{2 \pi}} \sqrt{\frac{\Delta_{r}-\Delta_{b}+\omega_{+}}{\Delta_{r}+\Delta_{b}+\omega_{+}}} \exp \left(-\frac{\Delta_{r}}{T}\right)
$$

As expected, the decay rate due to the first mechanism is exponentially suppressed due to the fact that it costs energy $\Delta$ to bring a quasiparticle from the normal parts.

The contribution of the second mechanism given by Eq. (12) depends on the density matrix of the box. The initial state of the qubit corresponds to the even-charge state in the CPB. Statistical weight of the states with even number of quasiparticles in the dot, $2,4,6, \ldots, 2 n$, is determined by the density matrix $\left.\rho_{2 n}\left(\beta H_{0}\right)\right)$,

$$
\left.\rho_{2 n}\left(\beta H_{0}\right)\right)=\operatorname{Tr}_{\{p\}} \rho\left(\beta H_{0}\right)=\frac{\exp \left(-\sum_{j=2}^{2 n} \frac{E_{k_{j}}}{T}\right)}{(2 n) ! Z_{\mathrm{ev}}},
$$

where $Z_{\mathrm{ev}}=\cosh \left(z_{b}\left(T, \delta_{b}\right)\right)$ is the partition function for the dot with even number of electrons ${ }^{18}$, and $z_{b}\left(T, \delta_{b}\right)$ is

$$
z_{b}\left(T, \delta_{b}\right)=\sum_{k} \exp \left(-\frac{E_{k}}{T}\right)=\sqrt{\frac{T}{\Delta_{b}}} \frac{\Delta_{b}}{\delta_{b}} \exp \left(-\frac{\Delta_{b}}{T}\right)
$$

According to Eq. (12) and Eq. (20), the contribution to the decay rate due to the second mechanism is obtained by summing over the states with even number of quasiparticles with appropriate statistical weight

$$
\begin{aligned}
\Gamma_{2}^{\mathrm{op}} & =2 \pi \sum_{n, p, k_{j}}\left|\left\langle N+1, p,\{k\}_{2 n-1}\left|H_{T}\right|+,\{k\}_{2 n}\right\rangle\right|^{2} \\
& \times \delta\left(E_{p}-E_{k_{1}}-\omega_{+}\right) 2 n \rho_{2 n}\left(\beta H_{0}\right)
\end{aligned}
$$

where, for example, $\left\langle N+1, p,\{k\}_{2 n-1}\right|$ is a state corresponding to the charge on the box equal to $1 e, 2 n-1$ quasiparticles in the box, and 1 quasiparticle in the reservoir

$\left|N+1, p,\{k\}_{2 n-1}\right\rangle=\left|N+1, k_{1}, \ldots, k_{j}, \ldots, k_{2 n-1}\right\rangle \otimes|p\rangle$.

The additional factor of $2 n$ in Eq. (22) is the result of the summation of $2 n$ identical terms in Eq. (12). The tunneling matrix element in Eq. (22) is determined using the particle-conserving Bogoliubov transformation and is dependent only on $p$ and $k_{1}$. Therefore, by doing the sum over the other momenta $k_{j}$, one gets the following result:

$$
\begin{aligned}
\Gamma_{2}^{\mathrm{op}} & =\pi \sum_{p, k_{1}}\left|\left\langle N+1, p\left|H_{T}\right| N+2, k_{1}\right\rangle\right|^{2} \delta\left(E_{p}-E_{k_{1}}-\omega_{+}\right) \\
& \times \exp \left(-\frac{E_{k_{1}}}{T}\right) \sum_{n} \frac{\left[z_{b}\left(T, \delta_{b}\right)\right]^{2 n-1}}{(2 n-1) ! Z_{\mathrm{ev}}}
\end{aligned}
$$

Changing the sum to an integral and integrating over $E_{p}$, we obtained the following expression:

$$
\begin{aligned}
\Gamma_{2}^{\mathrm{op}} & =\frac{g}{4 \pi} \int_{\Delta_{b}}^{\infty} d E_{k} \frac{\left(E_{k}\left(E_{k}+\omega_{+}\right)-\Delta_{r} \Delta_{b}\right)}{\sqrt{\left(\left(E_{k}+\omega_{+}\right)^{2}-\Delta_{r}^{2}\right)\left(E_{k}^{2}-\Delta_{b}^{2}\right)}} \\
& \times \Theta\left(E_{k}+\omega_{+}-\Delta_{r}\right) \exp \left(-\frac{E_{k}}{T}\right) \tanh \left(z_{b}\left(T, \delta_{b}\right)\right) .(25)
\end{aligned}
$$

The integration can be performed assuming that mismatch between superconducting gap energies in the box and reservoir is small, and $\Delta_{b}-\Delta_{r}+\omega_{+}>0$. Comparing Eqs. (17) and (25), one notices that the answer for $\Gamma_{2}^{\text {op }}$ can be expressed via $\Gamma_{1}^{\mathrm{op}}$ by permuting $\Delta_{r} \leftrightarrow \Delta_{b}$

$$
\Gamma_{2}^{\mathrm{op}}\left(\omega_{+}, \Delta_{r}, \Delta_{b}\right)=\Gamma_{1}^{\mathrm{op}}\left(\omega_{+}, \Delta_{b}, \Delta_{r}\right) \tanh \left(z_{b}\left(T, \delta_{b}\right)\right) .
$$

Taking into account Eq. (18) and Eq. (26), we find the 
total quasiparticle decay rate for the open system

$$
\begin{aligned}
\Gamma_{|N+1\rangle \leftarrow|+\rangle}^{\mathrm{op}} & =W\left(\omega_{+}, \Delta_{r}, \Delta_{b}\right)+W\left(\omega_{+}, \Delta_{b}, \Delta_{r}\right) \\
& \times \tanh \left(z_{b}\left(T, \delta_{b}\right)\right),
\end{aligned}
$$

where the first term corresponds to the first mechanism and is dominating for the open system. The simplified results for $\Gamma_{|N+1\rangle \leftarrow|+\rangle}^{\mathrm{op}}$ are discussed in Sec. IV.

Decay rate evaluation for the ground state of the qubit $|-\rangle$ can be done similarly provided that the final state $|N+1\rangle$ of the qubit is lower in energy than the initial state $|-\rangle$.

\section{STATES OF THE QUBIT IN AN ISOLATED SYSTEM}

\section{A. Thermodynamic properties of the qubit with fixed number of electrons}

When the number of electrons in the qubit is fixed, parity effects become important at low temperatures, $T<T_{r}^{*}, T_{b}^{*}$, where $T_{b}^{*}$ is defined in Eq. (3) and $T_{r}$ is equal to

$$
T_{r}^{*}=\frac{\Delta_{r}}{\ln \left(\Delta_{r} / \delta_{r}\right)} .
$$

Here, $\delta_{r}$ is the mean level spacing in the reservoir. The density of quasiparticles in the qubit with even number of electrons is small, $n \propto \exp (-2 \Delta / T)$, since at low temperatures all electrons are paired, and it costs energy $2 \Delta$ to break a Cooper pair. In the odd-charge state, an unpaired electron is present in the system even at zero temperature. It is important to understand the probability of finding a quasiparticle in the $\mathrm{CPB}$ since the qubit will not work if an extra electron is present in the box. To find out whether it is favorable or not for a quasiparticle to reside in the $\mathrm{CPB}$, we calculated the difference in free energy $\Delta F=F_{1}-F_{0}$ between two states: with and without a quasiparticle in the box ( $F_{1}$ and $F_{0}$, respectively). At the operating point, the free energy difference for the qubit with even $\left(\Delta F_{\text {ev }}\right)$ and odd $\left(\Delta F_{\text {odd }}\right)$ total number of electrons is given by the following expressions:

$$
\begin{aligned}
\Delta F_{\mathrm{ev}} & =-E_{c}+\frac{E_{J}}{2}-T \ln \left[\tanh \left(z_{b}\left(T, \delta_{b}\right)\right)\right] \\
& -T \ln \left[\tanh \left(z_{r}\left(T, \delta_{r}\right)\right)\right] \\
\Delta F_{\mathrm{odd}} & =-E_{c}+\frac{E_{J}}{2}-T \ln \left[\tanh \left(z_{b}\left(T, \delta_{b}\right)\right)\right] \\
& -T \ln \left[\operatorname{coth}\left(z_{r}\left(T, \delta_{r}\right)\right)\right]
\end{aligned}
$$

Negative value of $\Delta F$ indicates that free energy is lower for a quasiparticle in the CPB. Using these expressions, we can calculate thermodynamic probability $P(T)$ to find an unpaired electron in the box as a function of the physical parameters

$$
P(T)=\frac{Z_{1}}{Z_{1}+Z_{0}}=\frac{1}{\exp \left(\frac{\Delta F}{T}\right)+1},
$$

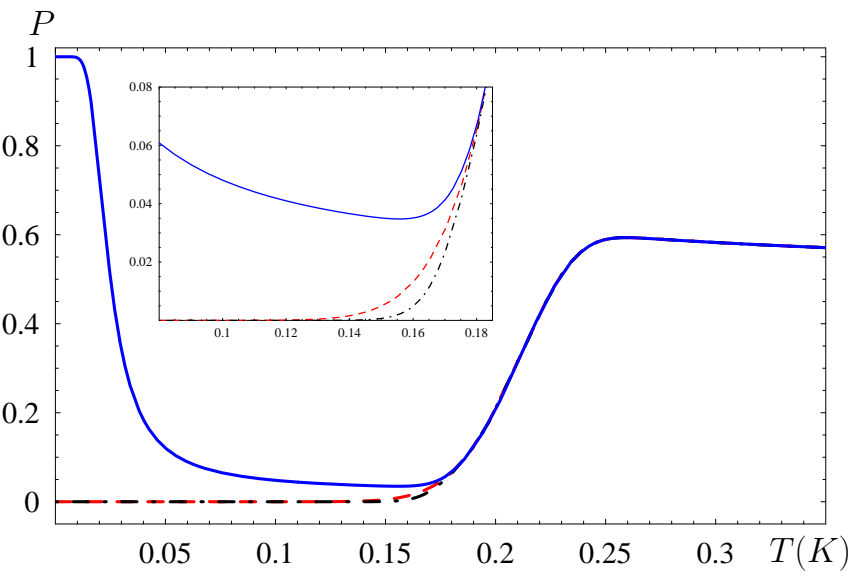

FIG. 2: (color online). Main panel: temperature dependence of the number of quasiparticles in the box at the operating point $\left(N_{g}=1\right)$. Dash-dot (black) line corresponds to even number of electrons, solid (blue) line - odd number of electrons, dash (red) line - open system. Physical parameters are chosen in correspondence to typical qubit experiments: $\Delta_{r}=\Delta_{b}=2.4 \mathrm{~K}, E_{c}=0.25 \mathrm{~K}, E_{J}=0.3 \mathrm{~K}, T_{b}^{*}=210 \mathrm{mK}$, and $T_{r}^{*}=160 \mathrm{mK}$ (see Eqs. (3) and (28) for definition of $T_{b}^{*}$ and $T_{r}^{*}$ ). Inset: temperature dependence of the number of quasiparticles in the $\mathrm{CPB}$ in the vicinity of $T_{r}^{*}$.

where $Z_{1(0)}=\exp \left(-\beta F_{1(0)}\right)$ is the partition function with one (zero) unpaired electrons in the box. The expression for the free energy difference $\Delta F_{o p}$ for the open system (fixed chemical potential regime) can be obtained using $\Delta F_{\text {ev }}$ and taking the limit of infinite volume of the reservoir $\left(\delta_{r} \rightarrow 0\right)$. Temperature dependence of the quasiparticle probability in the CPB for realistic parameters is plotted in Fig.2.

As shown in Fig.2, at high temperatures $T>T_{b}^{*}$ the probability of having an extra electron in the CPB coincides for an open and isolated qubit. At this temperature the number of thermal quasiparticles in the system is large, and parity effects are not important. Parity effects start to manifest themselves below the characteristic temperature $T_{b}^{*}$, when the number of quasiparticles in the box is of the order of unity. As can be seen from Fig.2, at the temperature $T_{r}^{*}$ the probability of having a quasiparticle in the CPB is negligible in the even-charge state, as well as in the open system. In the case of odd charge state of a qubit, lowering the temperature enhances quasiparticle poisoning 19 . This effect can be explained as a competition of two contributions to the free energy: a charging energy gained by tunneling to the box and entropy contribution proportional to the ratio of the volumes of the reservoir and box, $\sim V_{r} / V_{b}$. At the temperature $T_{s}$

$$
T_{s} \simeq \frac{E_{c}-E_{J} / 2}{\ln \left(\frac{V_{r}}{V_{b}}\right)}
$$

the entropy contribution becomes smaller, and the quasiparticle resides in the CPB. Thus, for the odd-charge state there is only a certain intermediate temperature 
range when qubit can work, i.e. can be prepared in the "good" quantum state. For physical parameters used in Fig.2 $T_{s}$ is approximately equal to $20 \mathrm{mK}$.

The presence of a quasiparticle in the box can be studied experimentally by measuring the periodicity of Coulomb staircase 11.12.13.14.15.17. According to the obtained results, the Coulomb staircase for an open system should be $2 e$ periodic below the temperature $\widetilde{T}_{b}^{*}$. The qubit with fixed number of electrons should have two distinct types of behavior corresponding to even and odd total number of electrons in the box and reservoir. In the former case the Coulomb staircase is similar to the one in the open system, while in the latter Coulomb staircase is $1 e$ periodic for temperatures above $T_{b}^{*}$, then $2 e$ periodic from $T_{b}^{*}$ to $T_{s}$, and again $1 e$ periodic for $T<T_{s}$.

In principle the probability to find a quasiparticle in the CPB can be lowered, and the qubit can be brought into the desired quantum state. We discuss several ways of doing this in Sec. IV. However, even if the quasiparticle is in the reservoir at the initial moment of time, once the qubit is excited and quantum oscillations emerge, the time of the oscillations is determined by the kinetics, i.e., by quasiparticle tunneling rate.

\section{B. Quasiparticle decay rate in the isolated system}

Let us turn to the discussion of the lifetime of the charge qubit in the regime with the fixed number of electrons. In order to calculate the quasiparticle decay rate, we proceed in the same manner as in the open system. The decay rate for the even number of electrons is calculated by averaging over initial states with even parity density matrix $\rho_{2 n}\left(\beta H_{0}\right)$. This situation corresponds to having an even number of electrons in the box and reservoir. The appearance of quasiparticles in the system occurs at the expense of breaking Cooper pairs. Using the results of analogous calculation in Eq. (26), we can write the expression for the total decay rate

$$
\begin{aligned}
\Gamma_{|N+1\rangle \leftarrow|+\rangle}^{\mathrm{ev}} & =W\left(\omega_{+}, \Delta_{r}, \Delta_{b}\right) \tanh \left(z_{r}\left(T, \delta_{r}\right)\right) \\
& +W\left(\omega_{+}, \Delta_{b}, \Delta_{r}\right) \tanh \left(z_{b}\left(T, \delta_{b}\right)\right),
\end{aligned}
$$

where $W\left(\omega_{+}, \Delta_{r}, \Delta_{b}\right)$ is defined in Eq. (18). The first term here corresponds to the first mechanism given by Eq. (111) and averaged over the even-parity initial state.

In the odd-charge case the decoherence rate is the largest since a quasiparticle is present in the system at $T=0$. Initial configuration of the system corresponds to having an odd number of quasiparticles in the reservoir. The reduced density matrix for this initial state $\rho_{2 n-1}\left(\beta H_{0}\right)$ is then given by

$$
\rho_{2 n-1}\left(\beta H_{0}\right)=\operatorname{Tr}_{\{k\}} \rho\left(\beta H_{0}\right)=\frac{\exp \left(-\sum_{j=1}^{2 n-1} \frac{E_{p_{j}}}{T}\right)}{(2 n-1) ! Z_{\text {odd }}},
$$

where $Z_{\text {odd }}=\sinh \left(z_{r}\left(T, \delta_{r}\right)\right)$. Using Eq. (11), we write the contribution to the decay rate of the first mechanism

$$
\begin{aligned}
\Gamma_{1}^{\text {odd }} & =2 \pi \sum_{n, p_{j}, k}\left|\left\langle N+1, k,\{p\}_{2 n-2}\left|H_{T}\right|+,\{p\}_{2 n-1}\right\rangle\right|^{2} \\
& \times \delta\left(E_{k}-E_{p_{1}}-\omega_{+}\right)(2 n-1) \rho_{2 n-1}\left(\beta H_{0}\right) .
\end{aligned}
$$

Going through the same arguments as in Eq. (24), the expression for $\Gamma_{1}^{\text {odd }}$ can be simplified

$$
\begin{aligned}
\Gamma_{1}^{\text {odd }} & =\pi \sum_{p_{1}, k}\left|\left\langle N+1, k\left|H_{T}\right| N, p_{1}\right\rangle\right|^{2} \delta\left(E_{k}-E_{p_{1}}-\omega_{+}\right) \\
& \times \exp \left(-\frac{E_{p_{1}}}{T}\right) \sum_{n=1}^{\infty} \frac{\left[z_{r}\left(T, \delta_{r}\right)\right]^{2 n-2}}{(2 n-2) ! Z_{\text {odd }}}
\end{aligned}
$$

Summing over $E_{k}$, we get

$$
\begin{aligned}
\Gamma_{1}^{\text {odd }} & =\frac{g}{4 \pi} \int_{\Delta_{r}}^{\infty} d E_{p} \frac{\left(E_{p}\left(E_{p}+\omega_{+}\right)-\Delta_{r} \Delta_{b}\right)}{\sqrt{\left(\left(E_{p}+\omega_{+}\right)^{2}-\Delta_{b}^{2}\right)\left(E_{p}^{2}-\Delta_{r}^{2}\right)}} \\
& \times \Theta\left(E_{p}+\omega_{+}-\Delta_{b}\right) \exp \left(-\frac{E_{p}}{T}\right) \operatorname{coth}\left(z_{r}\left(T, \delta_{r}\right)\right)(37)
\end{aligned}
$$

Taking into account Eq. (17) and Eq. (18), $\Gamma_{1}^{\text {odd }}$ is equal to

$$
\Gamma_{1}^{\text {odd }}=W\left(\omega_{+}, \Delta_{r}, \Delta_{b}\right) \operatorname{coth}\left(z_{r}\left(T, \delta_{r}\right)\right) .
$$

In order to find the contribution of the second mechanism, one has to average over initial states of the CPB. The initial configuration of the box corresponds to the even-charge state and is the same for open and isolated qubits. Therefore, contribution of the second mechanism, $\Gamma_{2}^{\text {odd }}$, is given by Eq. (26).

Total decay rate $\Gamma_{|N+1\rangle \leftarrow|+\rangle}^{\text {odd }}$ with odd number of electrons in the qubit is the sum of $\Gamma_{1}^{\text {odd }}$ and $\Gamma_{2}^{\text {odd }}$

$$
\begin{aligned}
\Gamma_{|N+1\rangle<+\rangle}^{\text {odd }} & =W\left(\omega_{+}, \Delta_{r}, \Delta_{b}\right) \operatorname{coth}\left(z_{r}\left(T, \delta_{r}\right)\right) \\
& +W\left(\omega_{+}, \Delta_{b}, \Delta_{r}\right) \tanh \left(z_{b}\left(T, \delta_{b}\right)\right)
\end{aligned}
$$

At low temperatures, $T<T_{r}^{*}, T_{b}^{*}$, the first term here is dominant as $z(T, \delta) \ll 1$. The detailed analysis of the low temperature asymtotics for different experimental regimes is presented below.

\section{DISCUSSION OF THE RESULTS}

Temperature dependence of the quasiparticle decay rate for different experimental realizations of the qubit is shown in Fig.3. As it is clear from the figure, at experimentally relevant temperatures $T \ll T_{r}^{*}, T_{b}^{*}$, the largest decay rate corresponds to the odd-charge case. In the vicinity of $T_{r}^{*}$ defined in Eq. (28), the decay rate is growing quickly due to the appearance of a large number of quasiparticles in the reservoir. As we approach the temperature $T=T_{b}^{*}$ given in Eq. (3), which corresponds to 


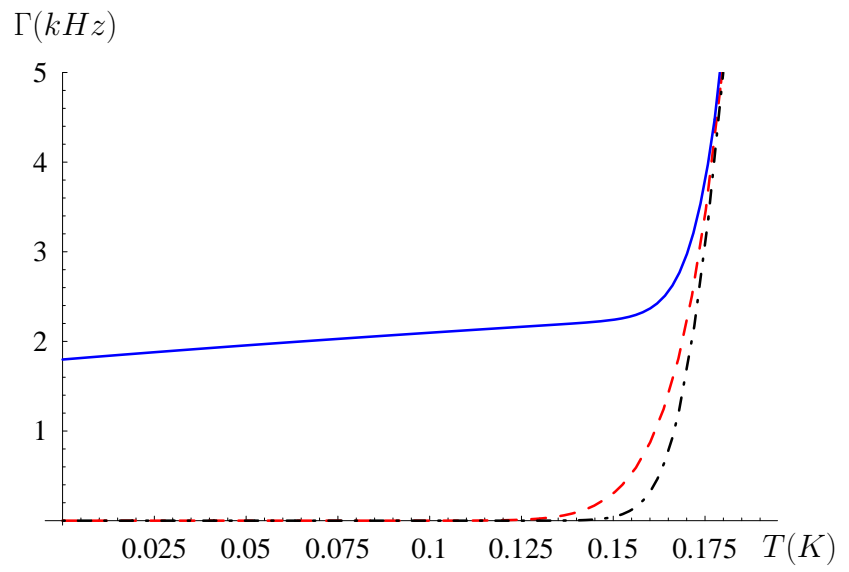

FIG. 3: (color online) Temperature dependence of the quasiparticle decay rate. Dash (red) line corresponds to the open system, dash-dot (black) - even number of electrons, solid (blue) - odd number of electrons. Here, we used the same physical parameters as specified in Fig. 2.

the appearance of quasiparticles in the Cooper pair box, parity effects become irrelevant and decoherence rates for different cases coincide.

Results obtained in Sec. II and III allow us to quantitatively estimate the decoherence rate due to the presence of quasiparticles in the system. For simplicity we assume that superconducting gap energies are the same in the box and reservoir, $\Delta_{b}=\Delta_{r}=\Delta$, and temperature is low, $T<T_{r}^{*}, T_{b}^{*} \ll \omega_{+}$, corresponding to typical qubit experiments $\left(\omega_{+}\right.$is defined in Eq. (2) ). In this approximation, for an "open" qubit the decay rate is

$$
\begin{aligned}
\Gamma_{|N+1\rangle \leftarrow|+\rangle}^{\mathrm{op}} \simeq & \frac{g \sqrt{T \Delta}}{8 \sqrt{\pi}} \sqrt{\frac{\omega_{+}}{\Delta+\omega_{+} / 2}} \exp \left(-\frac{\Delta}{T}\right) \\
& +\frac{g T}{8 \sqrt{\pi}} \sqrt{\frac{\omega_{+}}{\Delta+\omega_{+} / 2}} \frac{\Delta}{\delta_{b}} \exp \left(-\frac{2 \Delta}{T}\right) .
\end{aligned}
$$

The leading contribution to the decay rate for the open system $\Gamma_{|N+1\rangle \leftarrow|+\rangle}^{\mathrm{op}}$ is proportional to $\exp (-\Delta / T)$ since energy equal to $\Delta$ is required to bring an electron from the normal parts.

In the even-charge case the leading contribution to $\Gamma_{|N+1\rangle \leftarrow|+\rangle}^{\mathrm{ev}}$ is exponentially small, $\propto \exp (-2 \Delta / T)$, in accordance with the energy necessary to break a Cooper pair,

$$
\begin{aligned}
\Gamma_{|N+1\rangle \leftarrow|+\rangle}^{\mathrm{ev}} \simeq & \frac{g T}{8 \sqrt{\pi}} \sqrt{\frac{\omega_{+}}{\Delta+\omega_{+} / 2}} \frac{\Delta}{\delta_{r}} \exp \left(-\frac{2 \Delta}{T}\right) \\
& +\frac{g T}{8 \sqrt{\pi}} \sqrt{\frac{\omega_{+}}{\Delta+\omega_{+} / 2}} \frac{\Delta}{\delta_{b}} \exp \left(-\frac{2 \Delta}{T}\right) .
\end{aligned}
$$

Note that Eq. (41) and the last term in Eq. (40) are essentially the upper bounds for the contributions to the decay rate coming from unpaired electrons which originate in the isolated qubit and in the Cooper pair box, respectively. Indeed, we assumed in the derivation of Eqs. (40) and (41) that the decay rate is limited by the thermodynamic probability of the unpaired state, without discussing the kinetics of pair-breaking leading to such state. It is clear from Fig. 3, however, that the above mentioned contributions are negligibly small at low temperatures.

The decay rate of the qubit with an odd number of electrons $\Gamma_{|N+1\rangle \leftarrow|+\rangle}^{\text {odd }}$ is much larger

$$
\begin{aligned}
\Gamma_{|N+1\rangle \leftarrow|+\rangle}^{\mathrm{ddd}} & \simeq \frac{g \delta_{r}}{8 \sqrt{\pi}} \sqrt{\frac{\omega_{+}}{\Delta+\omega_{+} / 2}} \\
& +\frac{g T}{8 \sqrt{\pi}} \sqrt{\frac{\omega_{+}}{\Delta+\omega_{+} / 2}} \frac{\Delta}{\delta_{b}} \exp \left(-\frac{2 \Delta}{T}\right) .
\end{aligned}
$$

The leading contribution to the decay rate is temperature independent since the number of quasiparticles is finite even at $T=0$. According to the Eq. (42), the lifetime of qubit in the odd-charge case is determined by the conductance of the tunnel junctions and mean level spacing of the reservoir. For typical experiments quantum conductance, $g$ is of the order of one; $\delta_{r}$ depends on the volume of the reservoir and varies between $10^{-10}$ and $10^{-12} \mathrm{eV}$. With these parameters, decay rate $\Gamma_{|N+1\rangle \leftarrow|+\rangle}^{\text {odd }}$ can be estimated as $10^{5}-10^{3} \mathrm{~Hz}$ consequently. This is a substantial contribution to the decoherence of the isolated charge qubits, which limits qubit operation on a fundamental level. However, this decay rate is much smaller than the present estimates for decoherence in charge qubits; see, for example, the recent review by Devoret et. $a l^{6}$.

We assumed so far that in the initial state an unpaired electron resides in the reservoir and finally (after the relaxation) ends up in the box. Then, one can tune the qubit to the charge degeneracy between $1 e$ and $3 e$ and still have charge oscillations for some time until the quasiparticle escapes into the reservoir. In this case, the quasiparticle escape rate can be calculated in a similar way and is proportional to the conductance of the tunnel junction and level spacing in the Cooper-pair box: $\Gamma^{\text {odd }} \propto g \delta_{b}$.

In principle, quasiparticle poisoning can be decreased by tuning the proper parameters of the system such as superconducting gap energies $\Delta_{r, b}$, charging and Josephson energies, temperature, volumes of the box and reservoir. For example, it can be done by adjusting gap energies $\Delta_{r, b}$ with the help of oxygen doping $\frac{14}{}$ or magnetic field ${ }^{11}$. The latter is easy to implement since magnetic field is already used in charge qubits to tune Josephson energy $E_{J}$. In addition to the suppression of gap energies, large magnetic field $\left(H>H_{c 1}\right)$ can create vortices in the reservoir. The vortex acts as a quasiparticle trap since an unpaired electron gains gap energy $\Delta$ by residing inside the vortex.

\section{CONCLUSIONS}

We demonstrated that the presence of quasiparticles in the superconducting charge qubit leads to the decay of quantum oscillations. Two experimental realizations of 
charge qubit are considered here, corresponding to open and isolated system (in the former, the number of electrons is not fixed). Once the qubit is excited and quantum oscillations emerge, the decay of these oscillations is determined by the quasiparticle tunneling rate to the Cooper pair box. We calculated temperature dependence of the quasiparticle decay rate in the charge qubit. This decay rate is exponentially suppressed in the open system as well as in the isolated system with an even number of electrons. However, in the case with an odd number of electrons in the system, the quasiparticle decay rate is not exponentially suppressed and is estimated to be
$10^{5}-10^{3} \mathrm{~Hz}$ depending on the volume of the superconducting reservoir and conductance of the tunnel junctions.

\section{Acknowledgments}

We thank R. Schoelkopf and A. Wallraff for useful discussions. This work was supported by NSF grants DMR 02-37296, DMR 04-39026 and EIA 02-10736.
1 Y. Nakamura, Y. A. Pashkin, and J. S. Tsai, Nature 398, 786 (1999).

2 A. Wallraff, D. I. Schuster, A. Blais, L. Frunzio, R.-S. Huang, J. Majer, S. Kumar, S. M. Girvin, and R. J. Schoelkopf, Nature (London) 431, 162 (2004).

3 I. Chiorescu, Y. Nakamura, C. J. P. M. Harmans, and J. E. Mooij, Science 299, 1869 (2003).

${ }^{4}$ D. Vion, A. Aassime, A. Cottet, P. Joyez, H. Pothier, C. Urbina, D. Esteve, and M.H. Devoret, Science 296, 286 (2002).

5 A. Blais, R.-S. Huang, A. Wallraff, S. M. Girvin, and R. J. Schoelkopf, Phys. Rev. A 69, 062320 (2004)

6 M. H. Devoret, A. Wallraff, J. M. Martinis, cond-mat/0411174

7 M. T. Tuominen, J. M. Hergenrother, T. S. Tighe, and M. Tinkham, Phys. Rev. Lett. 69, 1997 (1992).

8 F. W. J. Hekking, L.I. Glazman, K. A. Matveev, and R. I. Shekhter, Phys. Rev. Lett. 70, 4138 (1993).

9 P. Lafarge, P. Joyez, D. Esteve, C. Urbina, and M.H. Devoret, Phys. Rev. Lett. 70, 994 (1993).

10 Y. Makhlin, G. Schoen, and A. Shnirman, Rev. Mod. Phys. 73, 357 (2001).
11 B. Turek, J. Majer, A. Clerk, S. Girvin, A. Wallraff, K. Bladh, D. Gunnarson, T. Duty, P. Delsing and R. Schoelkopf, Proceedings of Applied Superconductivity Conference, Jacksonville, FL 2004.

12 A. G. Guillaume, J. F. Schneiderman, P. Delsing, H. M. Bozler, and P. M. Echternach, Phys. Rev. B 69, 132504 (2004).

13 K. W. Lehnert, K. Bladh, L. F. Spietz, D. Gunnarson, D. I. Schuster, P. Delsing, and R. J. Schoelkopf, Phys. Rev. Lett. 90, 027002 (2003).

14 J. Aumentado, M. W. Keller, J. M. Martinis, M. H. Devoret, Phys. Rev. Lett. 92, 66802 (2004).

15 J. Mannik and J. E. Lukens, Phys. Rev. Lett. 92, 057004 (2004).

16 J.R. Schrieffer, Theory of Superconductivity, (Oxford : Advanced Book Program, Perseus, 1999).

17 V. Bouchiat, D. Vion, P. Joyez, D. Esteve, and M. H. Devoret, Physica Scripta, T76, 165 (1998).

18 K.A. Matveev, L.I. Glazman, and R.I. Shekhter Mod. Phys. Lett. B 8, 1007 (1994).

19 This effect was observed experimentally; see ref.[14] and references therein. 